American Journal of Applied Sciences 6 (6): 1143-1152, 2009

ISSN 1546-9239

(C) 2009 Science Publications

\title{
Phytoplankton Community of Elechi Creek, Niger Delta, Nigeria-A Nutrient-Polluted Tropical Creek
}

\author{
${ }^{1}$ O.A. Davies, ${ }^{2}$ J.F.N. Abowei and ${ }^{3}$ C.C. Tawari \\ ${ }^{1}$ Department of Fisheries and Aquatic Environment, Faculty of Agriculture, \\ Rivers State University of Science and Technology, Nkpolu, Port Harcourt, Nigeria \\ ${ }^{2}$ Department of Biological Sciences, Faculty of Science, \\ Niger Delta University, Wilberforce Island, Amassoma, Bayelsa State, Nigeria \\ ${ }^{3}$ Department of Fisheries and Livestock Production, Faculty of Agriculture, \\ Niger Delta University, Wilberforce Island, Amassoma, Bayelsa State, Nigeria
}

\begin{abstract}
Problem statement: Elechi creek of the upper bonny estuary in the Niger Delta contributes to the rivers state fish resources. It is a sink receiving organic anthropogenic wastes from Diobu, Eagle Island and waterfront dwellers of Diobu areas. Fishing, car washing, bathing, swimming and other human activities are constantly going on within and around this creek. Based on these activities, there is urgent need to study the phytoplankton community that supports its fisheries. Approach: The study investigated the phytoplankton composition, diversity, abundance and distribution as well as surface water physico-chemical parameters. Phytoplankton and surface water samples were collected bimonthly from October 2007-March 2008 at high tide from five stations according to APHA methods. These were analyzed for temperature, transparency, dissolved oxygen, salinity, alkalinity, chloride and nutrients. Phytoplankton was identified microscopically. Species diversity was calculated using standard indices. Results: A total of 169 species of phytoplankton, based on cell counts, was dominated by diatoms, 33255 counts $\mathrm{mL}^{-1}(36 \%)$ and blue-green algae, 32909 counts $\mathrm{mL}^{-1}(35.7 \%)$ were identified. The abundance of phytoplankton decreased downstream of this creek $(1>2>3>4)$ except in station 5 with the highest phytoplankton abundance (23938 counts $\mathrm{mL}^{-1}$ ). There was slight fluctuation in the measured physico-chemical parameters. The results of this study indicated the characteristic species and distribution of phytoplankton in Elechi Creek during the dry months. Conclusion/Recommendation: The high level of phosphate above the permissive limit showed that this creek is hypereutrophic and organic polluted. The high nutrients status favors the high abundance of phytoplankton. The municipal effluents (especially raw human and animal faces) discharges must be discontinued. Detergents with low concentration of phosphate are recommended for manufacturing and use. Municipal wastes must be treated and/or recycled before discharge into this natural aquatic body. Therefore, a continuous environmental surveillance of this creek is advocated to keep its biological integrity.
\end{abstract}

Key words: Diatoms, blue-green algae, species composition, hypereutropic, pollution

\section{INTRODUCTION}

The productivity of any water body is determined by the amount of plankton it contains as they are the major primary and secondary producers. Plankton communities serve as a base for the food chain that supports the commercial fisheries ${ }^{[1,2]}$. According to Wehr and Descy $^{[3]}$, phytoplankton communities are major producers of organic carbon in large rivers, a food source for planktonic consumers and may represents the primary oxygen source in many low-gradient rivers.
Phytoplankton are of great importance in biomonitoring of pollution. The distribution, abundance, species diversity, species composition of the phytoplankton are used to assess the biological integrity of the water body ${ }^{[1]}$. Also, they reflect the nutrient status of the environment. They do not have control over their movements thus they cannot escape pollution and this makes them a good indicator of pollution in the environment. Barnes ${ }^{[4]}$ reports that pollution affects plankton distribution, standing crop and chlorophyll concentration. This study was conducted to assess the

Corresponding Author: O.A. Davies, Department of Fisheries and Aquatic Environment, Faculty of Agriculture, Rivers State University of Science and Technology, Nkpolu, Port Harcourt, Nigeria 
characteristic phytoplankton species and their distribution in Elechi Creek. Also, it evaluated some surface water physico-chemical parameters.

\section{MATERIALS AND METHODS}

Study area: Elechi Creek, South-West of Port Harcourt metropolis, lies between longitude $6^{\circ} 45^{11} \mathrm{E}$ and $7^{\circ} 20^{\prime \prime} \mathrm{N}$ and latitude $4^{\circ} 38^{\prime \prime} \mathrm{N}$ and $5^{\circ} 5^{\prime \prime} \mathrm{E}$. The creek is a tributary of the upper limits of Bonny Estuary and includes its adjoining mangrove Creeks situated near the Eagle Island by the Rivers State University of Science and Technology, Nkpolu, Port Harcourt (Fig. 1). The vegetation is predominantly mangrove.

The low intertidal is dominated mostly by Rhizophora racemosa, $R$. mangle while the high intertidal is dominated by Avicennia africana, Laguncularia racemosa, Nypa fruticans and Aecrostichum aureum ${ }^{[5]}$. There are various fishing and transportation activities going on on Elechi Creek. Its vegetation provides logs of wood for domestic and building purposes. The area is also surrounded by numerous waterfront residential houses. The surrounding terrestrial environment is marked by various human activities such as saw milling of timber, free-range pig production, refuse dumping and car washing from Diobu and Eagle Island areas of Port Harcourt. Finally, the study area is a sink for numerous anthropogenic wastes from local industries.

Sampling stations: Samples were collected monthly for three months (October 2007-March 2008) from five sampling stations at high tide namely: (1) Waterfront residential buildings (Upstream), (2) UST brackish water fish pond, (3) A channel from UST female hostel, (4) Right timber market and (5) Left timber market (downstream) (Fig. 1). The six months samples were pooled according to sampling stations.

Sample collections and analyses: Field and laboratory measurements of some physico-chemical parameters of surface water were taken following standard methods ${ }^{[6]}$.

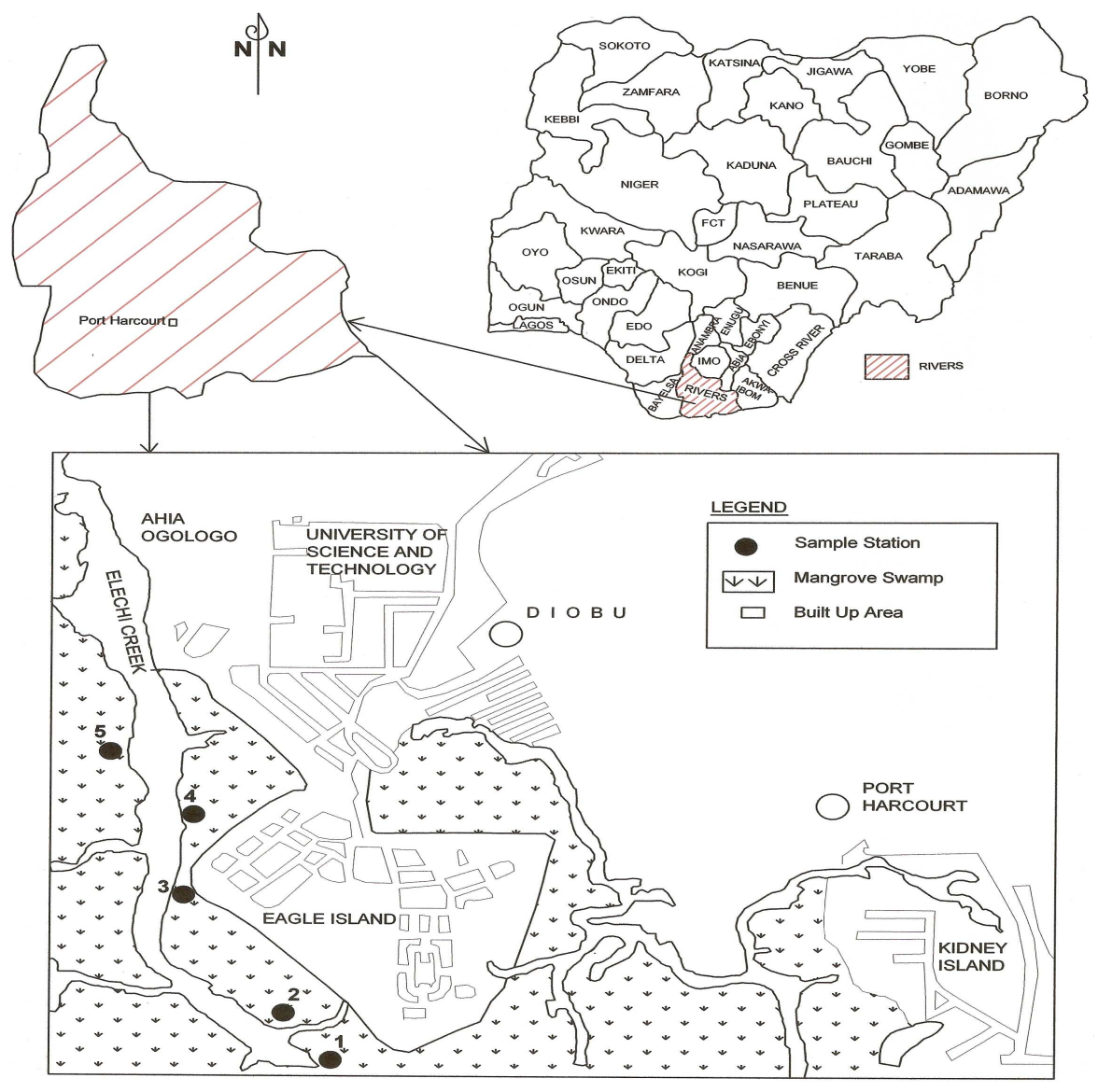

Fig. 1: Study area map 
Phytoplankton samples were collected by using sterilized, one-liter wide mouth plastic container at each sampling station ${ }^{[7]}$. The filtered samples were washed into the sterilized collecting bottles and immediately fixed in $4 \%$ formalin. Identification and enumeration were done by using leitzuezier binocular microscope and keys by Newell and Newell ${ }^{[8]}, \operatorname{Han}^{[9]}$, Prescott ${ }^{[10]}$ and Kadiri ${ }^{[11]}$.

\section{RESULTS}

Phytoplankton taxa: The recorded phytoplankton belonged to five taxa namely: Bacillariophyceae (diatoms), Cyanophyceae (blue-green algae), Euglenophyceae (euglenin), Chlorophyceae (green algae) and Dinophyceae (dinoflagellates). Diatoms $36.09 \%$ were the largest group of the phytoplankton and the least was dinoflagellates $0.02 \%$ (Table 1). One hundred and sixty nine species of phytoplankton were recorded. Phytoplankton abundance ranged between 13294 counts $\mathrm{mL}^{-1}$ (station 3 ) and 23938 counts $\mathrm{mL}^{-1}$ (station 5). A total of 108 species of diatoms were observed in the study stations (Table 2). The most dominant species was Navicula placentula (1167 counts $\mathrm{mL}^{-1}, 3.51 \%$ ) followed by Cyclotella comta (1099 counts $\left.\mathrm{mL}^{-1} 3.31 \%\right)$, Nitzschia sigma (1024 counts $\mathrm{mL}^{-1}, 3.08 \%$ ) and Melosira varians (1022 counts $\left.\mathrm{mL}^{-1}, 3.07 \%\right)$. The maximum number of species (108 species) was recorded in station 5 and the minimum (74 species) in station 1 . The number of bluegreen algae species ranged between 27 species (station 1) and 40 species (station 5) (Table 3). Anabaena spiroides (1712 counts $\mathrm{mL}^{-1}, 5.20 \%$ ) was the most abundant bluegreen algae species. Other prominent species were Anabaena flos-aquae (1657 counts $\left.\mathrm{mL}^{-1}, 5.04 \%\right)$, Oscillatoria limosa (1627 counts $\left.\mathrm{mL}^{-1}, 4.94 \%\right)$, Anabaena affinis (1568 counts $\mathrm{mL}, 4.77 \%)$ and Rivularia plancton $\left(1502\right.$ counts $\left.\mathrm{mL}^{-1}\right)$.

Table 1: Phytoplankton abundance in Elechi Creek

\begin{tabular}{|c|c|c|c|c|c|}
\hline Phytoplankton taxa & $\begin{array}{l}\text { Phytoplankton } \\
\text { abundance } \\
\text { (counts } \mathrm{mL}^{-1} \text { ) }\end{array}$ & $\begin{array}{l}\text { Percentage } \\
\text { abundance }(\%)\end{array}$ & No. of species & Station & $\begin{array}{l}\text { Phytoplankton } \\
\text { abundance } \\
\text { (counts } \mathrm{mL}^{-1} \text { ) }\end{array}$ \\
\hline Bacillariophyceae & 33255 & 36.09 & 108 & 1 & 20210 \\
\hline Cyanophyceae & 32909 & 35.72 & 40 & 2 & 19644 \\
\hline Euglenophyceae & 25868 & 28.08 & 9 & 3 & 15051 \\
\hline Chlorophyceae & 83 & 0.09 & 7 & 4 & 13294 \\
\hline Dinophyceae & 22 & 0.02 & 5 & 5 & 23938 \\
\hline Total & 92137 & 100.00 & 169 & 5 & 92137 \\
\hline
\end{tabular}

Table 2: Species composition and abundance of Bacillariophyceae in study stations

\begin{tabular}{|c|c|c|c|c|c|c|c|}
\hline S. No. & Bacillariophyceae species & Station 1 & Station 2 & Station 3 & Station 4 & Station 5 & Total \\
\hline 1 & Achnanthes sp.* & - & - & 4 & 6 & 17 & 27 \\
\hline 2 & Amphora ovalis & 50 & 84 & 55 & 56 & 63 & 308 \\
\hline 3 & Amphipleura pellucida & - & - & 5 & 6 & 13 & 24 \\
\hline 4 & Asterionella formosa & 48 & 82 & 23 & 24 & 28 & 205 \\
\hline 5 & A. gracillima & - & - & 1 & 3 & 10 & 14 \\
\hline 6 & Attheya zacharias & 41 & 75 & 46 & 48 & 55 & 265 \\
\hline 7 & Bacillaria* & - & 23 & 3 & 6 & 12 & 44 \\
\hline 8 & Bacteriastrum* & - & 34 & 5 & 8 & 15 & 62 \\
\hline 9 & Biddulphia* & - & 45 & 7 & 8 & 14 & 74 \\
\hline 10 & Cymbella affinis & 35 & 69 & 40 & 42 & 47 & 233 \\
\hline 11 & C. lacustris & 31 & 65 & 36 & 30 & 46 & 208 \\
\hline 12 & C. lanceolata & 35 & 69 & 40 & 43 & 50 & 237 \\
\hline 13 & C. amphioxys & 41 & 75 & 46 & 48 & 53 & 263 \\
\hline 14 & C. hybrida & 29 & 63 & 34 & 37 & 41 & 204 \\
\hline 15 & C. parva & 27 & 61 & 32 & 33 & 40 & 193 \\
\hline 16 & C. cistula & 42 & 73 & 40 & 45 & 51 & 251 \\
\hline 17 & C.tumida & 51 & 85 & 56 & 57 & 63 & 312 \\
\hline 18 & C. cuspidata & 45 & 79 & 50 & 53 & 58 & 285 \\
\hline 19 & C. lata & 48 & 82 & 53 & 55 & 62 & 300 \\
\hline 20 & Cyclotella antiqua & - & - & 2 & 5 & 11 & 18 \\
\hline 21 & C. comta & 211 & 245 & 211 & 213 & 219 & 1099 \\
\hline 22 & C. kutzingiana & - & - & - & 3 & 9 & 12 \\
\hline 23 & C. glomerata & 20 & 54 & 25 & 27 & 34 & 160 \\
\hline 24 & C. meneghiniana & 135 & 169 & 140 & 142 & 148 & 734 \\
\hline 25 & C. operculata & 153 & 187 & 158 & 160 & 165 & 823 \\
\hline 26 & C. striata & 30 & 61 & 32 & 34 & 40 & 197 \\
\hline 27 & Camphylodiscus hibernicus & - & 38 & 7 & 9 & 14 & 68 \\
\hline 28 & Cocconeis diminuta & 28 & 62 & 33 & 34 & 39 & 196 \\
\hline
\end{tabular}


Am. J. Applied Sci., 6 (6): 1143-1152, 2009

Table 2: Continued

\begin{tabular}{|c|c|c|c|c|c|c|c|}
\hline 29 & C. placentula & - & 63 & 6 & 7 & 12 & 88 \\
\hline 30 & C. scutellum & - & - & - & 3 & 8 & 11 \\
\hline 31 & Corethron hystrix & 170 & 204 & 175 & 177 & 156 & 882 \\
\hline 32 & Coscinodiscus excentricus & 42 & 76 & 47 & 49 & 54 & 268 \\
\hline 33 & C.lacustris & 33 & 67 & 38 & 39 & 47 & 224 \\
\hline 34 & C. radiata & 48 & 82 & 53 & 56 & 62 & 301 \\
\hline 35 & Cylindrotheca gracillis & - & - & - & 4 & 10 & 14 \\
\hline 36 & C. sp.* & - & - & - & 2 & 7 & 9 \\
\hline 37 & Cymptopleura elliptica & - & 30 & 3 & 4 & 11 & 48 \\
\hline 38 & Diatoma vulgare & 17 & 51 & 22 & 23 & 31 & 144 \\
\hline 39 & Diploneis elliptica & - & - & - & 3 & 8 & 11 \\
\hline 40 & Ditylum sp.* & - & - & - & 2 & 9 & 11 \\
\hline 41 & Epithemia argus & - & - & - & - & 1 & 1 \\
\hline 42 & E. turgida & - & - & - & - & 2 & 2 \\
\hline 43 & E. zebra & 39 & 73 & 44 & 46 & 51 & 253 \\
\hline 44 & Fragilaria capucina & 32 & 66 & 37 & 39 & 44 & 218 \\
\hline 45 & F. construens & 41 & 75 & 41 & 43 & 50 & 250 \\
\hline 46 & F. crotonesis & - & - & 2 & 4 & 10 & 16 \\
\hline 47 & $F$. intermedia & 25 & 59 & 30 & 32 & 38 & 184 \\
\hline 48 & F. sp.* & 26 & 60 & 31 & 34 & 40 & 191 \\
\hline 49 & F. virescens & - & - & - & 4 & 9 & 13 \\
\hline 50 & Frustulia rhomboides & 217 & 251 & 222 & 225 & 231 & 1146 \\
\hline 51 & Gomphonema acuminatum & 5 & 39 & 2 & 4 & 8 & 58 \\
\hline 52 & G. angustatum & - & - & 2 & 5 & 11 & 18 \\
\hline 53 & G. parvulum & 20 & 54 & 25 & 26 & 32 & 157 \\
\hline 54 & $G$ sp.* & - & - & 2 & 5 & 12 & 19 \\
\hline 55 & Gyrosigma acuminatum & 175 & 209 & 177 & 181 & 186 & 928 \\
\hline 56 & G. attenuatum & 167 & 201 & 172 & 175 & 182 & 897 \\
\hline 57 & G. paradox & 18 & 52 & 20 & 25 & 31 & 146 \\
\hline 58 & G. sp.* & - & 37 & 8 & 11 & 37 & 93 \\
\hline 59 & Hydrosera sp.* & - & 36 & 7 & 9 & 15 & 67 \\
\hline 60 & Melosira distans & 128 & 162 & 133 & 134 & 140 & 697 \\
\hline 61 & M. granulata & 34 & 68 & 39 & 41 & 46 & 228 \\
\hline 62 & M. japonica & - & - & 1 & 3 & 10 & 14 \\
\hline 63 & M. listans & 159 & 193 & 164 & 167 & 174 & 857 \\
\hline 64 & M. nummuloides & - & - & - & 2 & 8 & 10 \\
\hline 65 & M. pusilla & 160 & 184 & 155 & 157 & 163 & 819 \\
\hline 66 & $M$. sp.* & 37 & 71 & 42 & 43 & 49 & 242 \\
\hline 67 & M. undulata & 117 & 161 & 132 & 135 & 142 & 687 \\
\hline 68 & M. varians & 188 & 222 & 193 & 207 & 212 & 1022 \\
\hline 69 & Meridion sp.* & - & - & 3 & 6 & 14 & 23 \\
\hline 70 & Navicula amphibola & 159 & 188 & 159 & 160 & 166 & 832 \\
\hline 71 & N. bacillum & 170 & 199 & 170 & 173 & 181 & 893 \\
\hline 72 & N. cuspidata & 159 & 193 & 164 & 167 & 173 & 856 \\
\hline 73 & N. gracilis & 55 & 79 & 50 & 51 & 58 & 293 \\
\hline 74 & N. microcephala & 167 & 206 & 177 & 178 & 182 & 910 \\
\hline 75 & N. placentula & 233 & 225 & 233 & 235 & 241 & 1167 \\
\hline 76 & Nitzschia bilobata & 147 & 181 & 152 & 153 & 185 & 791 \\
\hline 77 & N. filiforms & 185 & 214 & 185 & 186 & 191 & 961 \\
\hline 78 & N. lanceolata & 64 & 98 & 69 & 71 & 78 & 380 \\
\hline 79 & $N$. linearis & 20 & 61 & 32 & 34 & 39 & 186 \\
\hline 80 & N. longissima & 178 & 207 & 178 & 181 & 188 & 932 \\
\hline 81 & N. paradoxa & 52 & 92 & 63 & 65 & 71 & 343 \\
\hline 82 & N. sigma & 189 & 228 & 199 & 201 & 207 & 1024 \\
\hline 83 & Pinnularia hemiptera & 9 & 43 & 14 & 15 & 21 & 102 \\
\hline 84 & P. horealis & 5 & 39 & 10 & 11 & 15 & 80 \\
\hline 85 & P. macilenta & 15 & 49 & 5 & 8 & 12 & 89 \\
\hline 86 & P. major & 7 & 71 & 42 & 44 & 52 & 216 \\
\hline 87 & P. mesolepta & 17 & 51 & 22 & 24 & 30 & 144 \\
\hline 88 & P. viridis & 13 & 47 & 18 & 21 & 27 & 126 \\
\hline 89 & Rhizolenia eriensis & - & - & - & 5 & 6 & 11 \\
\hline 90 & R. longiseta & 37 & 71 & 42 & 45 & 51 & 246 \\
\hline 91 & Skeletonema sp.* & - & - & 1 & 3 & 8 & 12 \\
\hline 92 & Stauroneis acuta & 45 & 79 & 50 & 51 & 57 & 282 \\
\hline 93 & S. parvula & 56 & 90 & 61 & 64 & 68 & 339 \\
\hline
\end{tabular}


Am. J. Applied Sci., 6 (6): 1143-1152, 2009

Table 2: Continued

\begin{tabular}{|c|c|c|c|c|c|c|c|}
\hline 94 & Stephanodiscus astrae & 29 & 60 & 31 & 32 & 38 & 190 \\
\hline 95 & S. sp.* & - & 5 & 4 & 6 & 13 & 28 \\
\hline 96 & Synedra acus & 80 & 114 & 85 & 86 & 92 & 457 \\
\hline 97 & S. affinis & 171 & 205 & 176 & 178 & 182 & 912 \\
\hline 98 & S. ulna & 201 & 236 & 206 & 207 & 215 & 1064 \\
\hline 99 & Surirella elegans & 21 & 55 & 26 & 29 & 36 & 167 \\
\hline 100 & S. robusta & 15 & 49 & 20 & 23 & 29 & 136 \\
\hline 101 & S. tenera & 38 & 72 & 43 & 44 & 49 & 246 \\
\hline 102 & S. spiralis & 24 & 58 & 29 & 31 & 37 & 179 \\
\hline 103 & S. sp.* & - & - & - & - & 1 & 1 \\
\hline 104 & Tabellaria binalis & - & - & - & - & 1 & 1 \\
\hline 105 & T. fenestrata & 49 & 83 & 54 & 58 & 62 & 306 \\
\hline 106 & T. flocculosa & 35 & 69 & 30 & 31 & 37 & 202 \\
\hline 107 & Thalossiothrix longissimum & - & 32 & 3 & 5 & 13 & 53 \\
\hline \multirow[t]{3}{*}{108} & T. sp.* & - & - & - & 4 & 11 & 15 \\
\hline & Total (counts $\mathrm{mL}^{-1}$ ) & 5643 & 8474 & 6015 & 6247 & 6876 & 33255 \\
\hline & No. of species & 74 & 84 & 94 & 104 & 108 & \\
\hline
\end{tabular}

-: Absent; *: Unidentified species; No: Number; S/N: Serial number

Table 3: Species composition and abundance of Cyanophyceae in study station

\begin{tabular}{|c|c|c|c|c|c|c|c|}
\hline S. No. & Cyanophyceae species & Station 1 & Station 2 & Station 3 & Station 4 & Station 5 & Total \\
\hline 1 & Anabaena affinis & 734 & 351 & 185 & 150 & 148 & 1568 \\
\hline 2 & A. circirolis & - & 180 & 136 & 101 & 35 & 452 \\
\hline 3 & A. sp.* & - & 210 & 191 & 156 & 90 & 647 \\
\hline 4 & A. spiroides & 827 & 406 & 197 & 162 & 120 & 1712 \\
\hline 5 & A. flos-aquae & 826 & 361 & 194 & 159 & 117 & 1657 \\
\hline 6 & Anabaenopsis arnoldii & 617 & 376 & 184 & 149 & 107 & 1433 \\
\hline 7 & Anacystis sp.* & 615 & 381 & 186 & 151 & 109 & 1442 \\
\hline 8 & Aphanothece stagnina & - & - & 113 & 38 & 32 & 183 \\
\hline 9 & A. clathrata & - & - & - & 40 & 30 & 70 \\
\hline 10 & Cochochloris stagnina & - & - & 190 & 155 & 89 & 434 \\
\hline 11 & Lyngbya contoria & - & - & 155 & 120 & 54 & 329 \\
\hline 12 & L. limtica & 170 & 105 & 153 & 118 & 52 & 598 \\
\hline 13 & L. major & 182 & 98 & 134 & 99 & 33 & 546 \\
\hline 14 & Merismopedia $s p^{*}$ & - & - & - & - & 30 & 30 \\
\hline 15 & Microcystis aeruginosa & 125 & 205 & 176 & 141 & 75 & 722 \\
\hline 16 & M. flos-aquae & 100 & 176 & 187 & 152 & 86 & 701 \\
\hline 17 & M. grevillei & 426 & 170 & 118 & 83 & 52 & 849 \\
\hline 18 & M. pulverea & 502 & 190 & 173 & 138 & 72 & 1075 \\
\hline 19 & Mougectia sp.* & - & - & 172 & 137 & 71 & 380 \\
\hline 20 & Nodularia sp.* & - & 210 & 180 & 145 & 81 & 616 \\
\hline 21 & Nostoc planctonicum & - & 160 & 138 & 103 & 67 & 468 \\
\hline 22 & $N$. verrucosum & 130 & 225 & 144 & 109 & 43 & 651 \\
\hline 23 & Oscillatoria lacustris & 572 & 411 & 193 & 158 & 116 & 1450 \\
\hline 24 & O. limosa & 734 & 413 & 199 & 160 & 121 & 1627 \\
\hline 25 & O.princeps & 447 & 150 & 145 & 110 & 60 & 912 \\
\hline 26 & O. rubescens & 150 & 112 & 197 & 162 & 96 & 717 \\
\hline 27 & O. tenuis & 500 & 391 & 180 & 145 & 103 & 1319 \\
\hline 28 & Phormidium mисіola & 201 & 117 & 122 & 87 & 21 & 548 \\
\hline 29 & P. sp.* & - & 192 & 123 & 88 & 45 & 448 \\
\hline 30 & P. tenиe & 445 & 219 & 111 & 76 & 79 & 930 \\
\hline 31 & P. valderiae & - & - & - & - & 39 & 39 \\
\hline 32 & Raphidiopisis curvata & 122 & 140 & 120 & 85 & 88 & 555 \\
\hline 33 & R. mediteranea & - & - & 143 & 108 & 77 & 328 \\
\hline 34 & Rivularia plancton & 600 & 440 & 195 & 160 & 107 & 1502 \\
\hline 35 & Spirulina laxissima & 590 & 378 & 198 & 163 & 121 & 1450 \\
\hline 36 & S. major & 156 & 130 & 150 & 115 & 87 & 638 \\
\hline 37 & S.princeps & 190 & 150 & 153 & 118 & 54 & 665 \\
\hline 38 & S. subtilissima & 425 & 147 & 148 & 113 & 37 & 870 \\
\hline 39 & Tolypothris distorta & 582 & 363 & 196 & 161 & 119 & 1421 \\
\hline \multirow[t]{3}{*}{40} & Trichodes lacastre & 451 & $160^{\circ}$ & 139 & 104 & 73 & 927 \\
\hline & Total (counts $\mathrm{mL}^{-1}$ ) & 11419 & 7717 & 6018 & 4719 & 3036 & 32909 \\
\hline & No. of species & 27 & 32 & 37 & 38 & 40 & \\
\hline
\end{tabular}

-: Absent; *: Unidentified species; No: Number; S/N: Serial number 
Am. J. Applied Sci., 6 (6): 1143-1152, 2009

Table 4: Euglenophyceae species composition and abundance in study stations

\begin{tabular}{|c|c|c|c|c|c|c|c|}
\hline S. No. & Euglenophyceae species & Station 1 & Station 2 & Station 3 & Station 4 & Station 5 & Total \\
\hline 1 & Euglena acus & 616 & 853 & 655 & 686 & 3067 & 5877 \\
\hline 2 & E. convoluta & 290 & 323 & 280 & 208 & 2576 & 3677 \\
\hline 3 & E. gracilis & 606 & 731 & 641 & 748 & 3082 & 5808 \\
\hline 4 & E. oxyuris & 115 & - & - & - & - & 115 \\
\hline 5 & E. viridis & 374 & 407 & 360 & 410 & 2744 & 4295 \\
\hline 6 & E. wangi & 362 & 393 & 352 & - & - & 1107 \\
\hline 7 & Lepocinclis ovata & 334 & 367 & 324 & 252 & - & 1277 \\
\hline 8 & Phacus acuminatus & 200 & 378 & 404 & - & - & 982 \\
\hline \multirow[t]{3}{*}{9} & Trachelomona cylindrica & 224 & - & - & - & 2506 & 2730 \\
\hline & Total (counts $\mathrm{mL}^{-1}$ ) & 3121 & 3452 & 3016 & 2304 & 13975 & 25868 \\
\hline & No. of species & 9 & 8 & 7 & 5 & 5 & \\
\hline
\end{tabular}

-: Absent; *: Unidentified species; No: Number; S/N: Serial number

Table 5: Species composition and abundance of Chlorophyceae in study stations

\begin{tabular}{|c|c|c|c|c|c|c|c|}
\hline S. No. & Chlorophyceae species & Station 1 & Station 2 & Station 3 & Station 4 & Station 5 & Total \\
\hline 1 & Ankistrodesmus falcatus & - & - & - & 1 & 3 & 4 \\
\hline 2 & Cosmarium granatum & 9 & - & - & 3 & 8 & 20 \\
\hline 3 & Pithiphora sp.* & - & - & - & - & 2 & 2 \\
\hline 4 & Scenedesmus acuminatus & 11 & 1 & 2 & 6 & 13 & 33 \\
\hline 5 & Selenastrum sp.* & - & - & - & 2 & 4 & 6 \\
\hline 6 & Stichococcus sp.* & - & - & - & - & 3 & 3 \\
\hline \multirow[t]{3}{*}{7} & Ulothrix sp* & 7 & - & - & 3 & 5 & 15 \\
\hline & Total (counts $\mathrm{mL}^{-1}$ ) & 27 & 1 & 2 & 15 & 38 & 83 \\
\hline & No. of species & 3 & 1 & 1 & 5 & 7 & \\
\hline
\end{tabular}

-: Absent; *: Unidentified species; No: Number; S/N: Serial number

Table 6: Species composition and abundance of Dinophyceae in study station

\begin{tabular}{llllllll}
\hline S. No. & Dinophyceae species & Station 1 & Station 2 & Station 3 & Station 4 & Station 5 & Total \\
\hline 1 & Ceratium furcas & - & - & - & 3 & 4 & 7 \\
2 & Gymnodinium aeruginosum & - & - & - & - & 1 \\
3 & Peridinium cinatum & - & - & - & 1 & 2 \\
4 & P. hirudinella & - & - & - & 4 & 5 \\
5 & P. umbonatum & - & - & - & 1 & 9 \\
\hline & Total (counts $\mathrm{mL}^{-1}$ ) & - & - & - & 9 & 13 & 2 \\
\hline & No. of species & - & - & - & 4 & 5 \\
\hline
\end{tabular}

-: Absent; *: Unidentified species; No: Number; S/N: Serial number

The maximum number of species of euglenin (9 species) was recorded in station 1 and the minimum (5 species) in stations 4 and 5 respectively (Table 4). Euglena acus (5877 counts $\mathrm{mL}^{-1}, 22.72 \%$ ) was the most abundant euglenin. Generally, Euglena species had the highest abundance of the Euglenophyceae. The number of green algae ranged from 1 species (stations 2 and 3) to 7 species (station 3). Scenedesmus acuminatus (33 counts $\mathrm{mL}^{-1}, 39.76 \%$ ) was the most abundant species (Table 5). Dinoflagellates were absent in stations 1-3. Only 5 species dominated by Peridinium hirudinella (9 counts $\mathrm{mL}^{-1}, 40.9 \%$ ) were recorded (Table 6).

Species diversity: From Table 7, Bacillariophyceae was the most diversified phytoplankton in terms of Margalef species richness (d), Shannon $\left(\mathrm{H}^{1}\right)$, Evenness $\left(E^{1}\right)$ and Dominance indices (D). The highest species richness of diatoms 12.11 was recorded in Station 5 and the lowest 8.45 in station 1. Generally, the dominance index was low (less than 1) for all taxa.

For Cyanophyceae, station 5 recorded the maximum $\mathrm{d} 4.86$ and station 1 the minimum $2.78 . \mathrm{H}^{1}$ ranged between 3.06 (station 1) and 3.62 (station 5). The highest d 0.99 for Euglenophyceae was observed in station 1 and the lowest d 0.42 in station 5 while station 1 recorded the maximum $\mathrm{H}^{1} 2.09$ and station 4 the maximum 1.49. Stations 2 and 3 recorded zero species diversity indices for Chlorophyceae. In other stations, the observed diversity indices were $\mathrm{d}, 0.61$ (station 1) and 1.65 (station 5) and $\mathrm{H}^{1} 1.08$ (station 1) and 1.76 (station 5). Dinophyceae were absent in stations 1,2 and 3 hence zero species diversity were recorded. Station $5 \mathrm{~d} 1.56$ and $\mathrm{H}^{1} 1.41$ were higher than $\mathrm{d} 1.37$ and $\mathrm{H}^{1} 1.22$ of station 4 but an opposite trend was observed for $\mathrm{E}^{1}$ and $\mathrm{D}$. 
Am. J. Applied Sci., 6 (6): 1143-1152, 2009

Table 7: Phytoplankton species diversity indices in the study stations

\begin{tabular}{|c|c|c|c|c|c|c|}
\hline Plankton taxa & Species diversity index & Station 1 & Station 2 & Station 3 & Station 4 & Station 5 \\
\hline \multirow[t]{4}{*}{ Bacillariophyceae } & $\mathrm{d}$ & 8.45 & 9.18 & 10.69 & 11.79 & 12.11 \\
\hline & $\mathrm{H}^{1}$ & 4.49 & 5.02 & 4.82 & 4.85 & 4.89 \\
\hline & $E^{1}$ & 2.08 & 2.21 & 2.15 & 2.12 & 2.09 \\
\hline & D & 0.02 & 0.05 & 0.03 & 0.03 & 0.04 \\
\hline \multirow[t]{4}{*}{ Cyanophyceae } & $\mathrm{d}$ & 2.78 & 3.46 & 4.14 & 4.37 & 4.86 \\
\hline & $\mathrm{H}^{1}$ & 3.06 & 3.46 & 3.55 & 3.59 & 3.62 \\
\hline & $E^{1}$ & 0.93 & 0.95 & 0.97 & 0.99 & 1.03 \\
\hline & D & 0.04 & 0.03 & 0.03 & 0.03 & 0.02 \\
\hline \multirow[t]{4}{*}{ Euglenophyceae } & $\mathrm{d}$ & 0.99 & 0.74 & 0.75 & 0.52 & 0.42 \\
\hline & $\mathrm{H}^{1}$ & 2.09 & 1.88 & 1.89 & 1.49 & 1.61 \\
\hline & $E^{1}$ & 0.95 & 0.97 & 0.97 & 0.93 & 1.00 \\
\hline & $\mathrm{D}$ & 0.14 & 0.17 & 0.16 & 0.23 & 0.20 \\
\hline \multirow[t]{4}{*}{ Chlorophyceae } & $\mathrm{d}$ & 0.61 & 0.00 & 0.00 & 1.48 & 1.65 \\
\hline & $\mathrm{H}^{1}$ & 1.08 & 0.00 & 0.00 & 1.46 & 1.76 \\
\hline & $E^{1}$ & 0.98 & 0.00 & 0.00 & 0.91 & 0.90 \\
\hline & $\mathrm{D}$ & 0.35 & 0.00 & 0.00 & 0.21 & 0.18 \\
\hline \multirow[t]{4}{*}{ Dinophyceae } & $\mathrm{d}$ & 0.00 & 0.00 & 0.00 & 1.37 & 1.56 \\
\hline & $\mathrm{H}^{1}$ & 0.00 & 0.00 & 0.00 & 1.22 & 1.41 \\
\hline & $\mathrm{E}^{\mathrm{l}}$ & 0.00 & 0.00 & 0.00 & 0.88 & 0.55 \\
\hline & $\mathrm{D}$ & 0.00 & 0.00 & 0.00 & 0.25 & 0.22 \\
\hline
\end{tabular}

d: Margalef species richness; $\mathrm{H}^{1}$ : Shannon index; El: Evenness index; D: Simpson dominance index

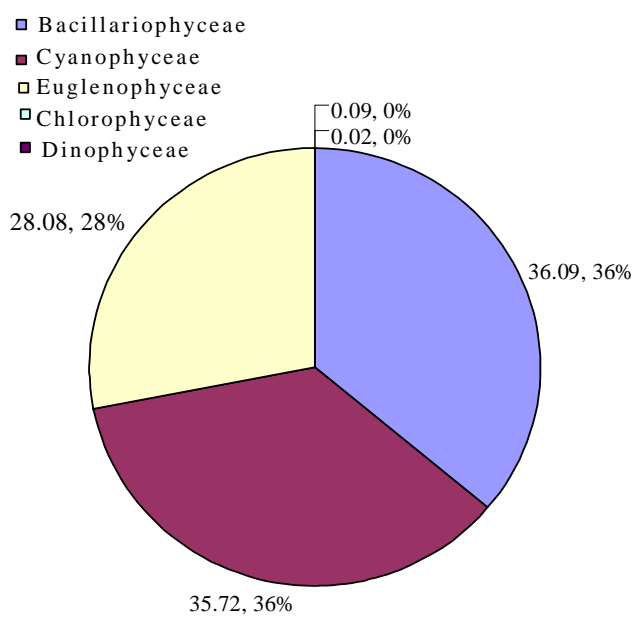

Fig. 2: Percentage contribution of phytoplankton groups in Elechi Creek

Phytoplankton contribution in Elechi Creek: Bacillariophyceae contributed the highest number of phytoplankton $36.09 \%$ in Elechi Creek followed by Cyanophyceae $35.72 \%$ and the lowest was Dinophyceae $0.02 \%$ (Fig. 2). From Fig. 3, the order of diatoms in the stations was $2>5>4>3>1$. For Cyanophyceae, it was $1>2>3>4>5$, for Euglenophyceae, the order was $5>2>1>3>4$ and for Chlorophyceae, it was $3>1>4>3>1$. Stations 4 and 5 had the same percentage contribution for Dinophyceae.

Physico-chemical parameters: Temperature variation in relation to station was insignificant $(\mathrm{p}>0.05)$ and ranged between $29.0^{\circ} \mathrm{C}$ (Station 5) and $30.7^{\circ} \mathrm{C}$

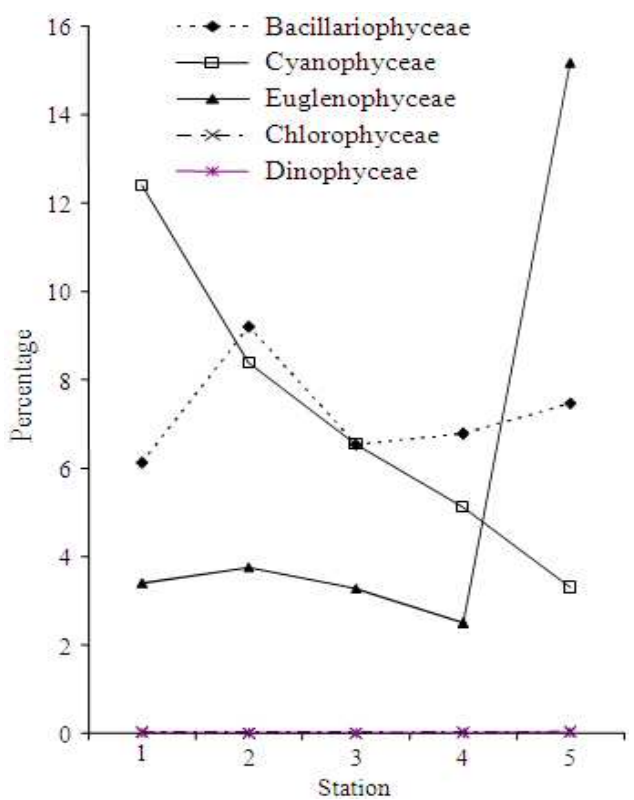

Fig. 3: Spatial percentage contribution of phytoplankton group in Elechi Creek

(station 1) (Table 8). The lowest transparency $(0.27 \mathrm{~m})$ was recorded in station 4 and the highest $0.67 \mathrm{~m}$ in station 2. Salinity ranged from $7.36 \%$ (station 4) to $22.73 \%$ (station 5). The maximum chloride $8430 \mathrm{mg} \mathrm{L}^{-1}$ was observed in Station 5 and minimum $2832 \mathrm{mg} \mathrm{L}^{-1}$ in station 4 . The alkalinity ranged between $48 \mathrm{mg} \mathrm{L}^{-1}$ (station 1) and $80 \mathrm{mg} \mathrm{L}^{-1}$ (station 4). Sulphate was highest in station $11231.3 \mathrm{mg} \mathrm{L}^{-1}$ and lowest in station 4 (377.6 mg L ${ }^{-1}$ ). 
Am. J. Applied Sci., 6 (6): 1143-1152, 2009

Table 8: Physico-chemical quality of surface water in the study stations

\begin{tabular}{|c|c|c|c|c|c|}
\hline Parameter & Station 1 & Station 2 & Station 3 & Station 4 & Station 5 \\
\hline Temperature $\left({ }^{\circ} \mathrm{C}\right)$ & $30.70 \pm 0.03^{\mathrm{a}}$ & $3.03 \pm 0.04^{\mathrm{a}}$ & $29.70 \pm 0.01^{\mathrm{a}}$ & $29.00 \pm 0.01^{\mathrm{a}}$ & $29.30 \pm 0.02^{\mathrm{a}}$ \\
\hline Transparency (m) & $0.48 \pm 0.01^{\mathrm{b}}$ & $0.67 \pm 0.02^{\mathrm{a}}$ & $0.59 \pm 0.02^{\mathrm{b}}$ & $0.27 \pm 0.03^{\mathrm{c}}$ & $0.75 \pm 0.10^{\mathrm{a}}$ \\
\hline Salinity $(\% o)$ & $20.50 \pm 0.83^{\mathrm{a}}$ & $18.50 \pm 1.60^{\mathrm{b}}$ & $1.27 \pm 1.22^{\mathrm{c}}$ & $7.36 \pm .0 .10^{\mathrm{d}}$ & $22.73 \pm 1.83^{\mathrm{a}}$ \\
\hline Chloride $\left(\mathrm{mg} \mathrm{L}^{-1}\right)$ & $69820.00 \pm 63.79^{b}$ & $6059.00 \pm 60.50^{\mathrm{b}}$ & $4017.00 \pm 46.41^{\mathrm{c}}$ & $2832.00 \pm 20.62^{\mathrm{d}}$ & $8430.00 \pm 80.19^{\mathrm{a}}$ \\
\hline Alkalinity $\left(\mathrm{mg} \mathrm{L}^{-1}\right)$ & $48.00 \pm 0.75^{\mathrm{c}}$ & $50.00 \pm 1.92^{\mathrm{c}}$ & $56.70 \pm 3.94^{\mathrm{b}}$ & $80.00 \pm 3.94^{\mathrm{a}}$ & $52.00 \pm 2.78^{\mathrm{c}}$ \\
\hline Sulphate $\left(\mathrm{mg} \mathrm{L}^{-1}\right)$ & $1231.30 \pm 101.01^{\mathrm{a}}$ & $767.00 \pm 42.77^{\mathrm{b}}$ & $584.70 \pm 42.85^{\mathrm{c}}$ & $377.60 \pm 43.86^{\mathrm{d}}$ & $1147.20 \pm 90.26^{\mathrm{a}}$ \\
\hline
\end{tabular}

Means in the same row with the same letter are not significantly different ( $p>0.05)$

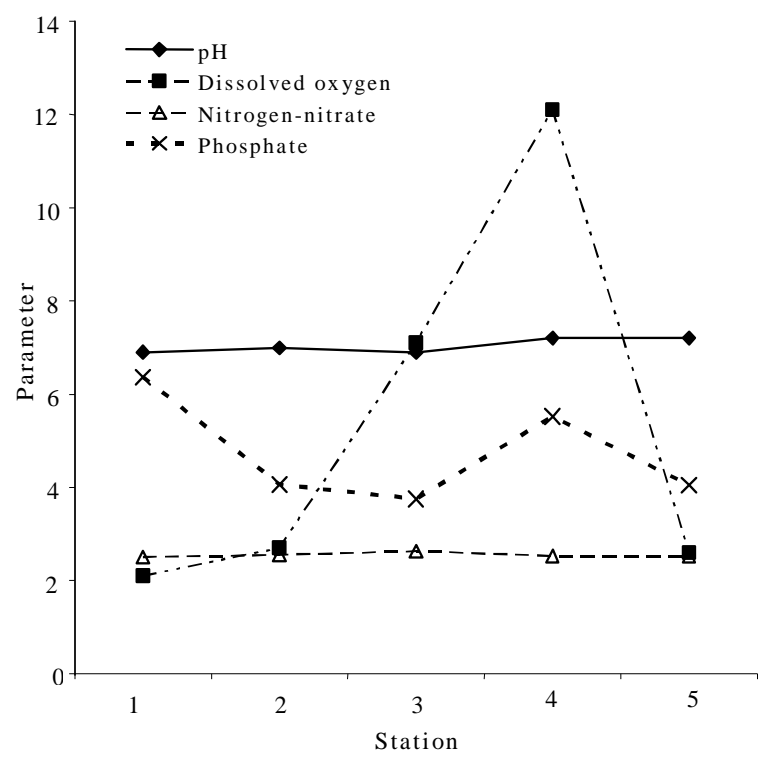

Fig. 4: Chemical quality of surface water in the study stations

$\mathrm{pH}$ values was maximum 7.2 in station 4 and minimum 6.9 in station 1 (Fig. 4). The highest dissolved oxygen $12.1 \mathrm{mg} \mathrm{L}^{-1}$ was recorded in station 4 and lowest $2.1 \mathrm{mg} \mathrm{L}^{-1}$ in station 1 . Nitrogen-nitrate ranged between $2.50 \mathrm{mg} \mathrm{L}^{-1}$ station 1 and $2.63 \mathrm{mg} \mathrm{L}^{-1}$ (station 3). The highest phosphate level $6.36 \mathrm{mg} \mathrm{L}^{-1}$ was observed in station 1 and the lowest $3.75 \mathrm{mg} \mathrm{L}^{-1}$ in station 3.

\section{DISCUSSION}

The high phytoplankton species composition, diversity and abundance recorded for the entire study were more than the values reported for studies in other waters of Bonny Estuary and Niger Delta. This indicates that these phytoplankton will support commercial fisheries in this creek $^{[1,2]}$. This might be attributed to the high nutrients status (phosphate, nitrate and sulphate). This creek receives enormous quantities of anthropogenic wastes (domestic and industries) such as raw human and animal faces from its surroundings.
These wastes increase the nutrients capabilities of this creek. The present observation might be attributed to environmental influence like high temperature, low $\mathrm{pH}$, transparency and dissolved oxygen. Phosphorus stimulates phytoplankton (algae) growth. According to Frankovick et al. ${ }^{[12]}$, the epiphytic diatom assemblage of the Florida Bay Estuary was structured by nutrient availability particularly phosphorus Phosphate might have structured the phytoplankton community of Elechi Creek. High temperature enhances photosynthesis and this is expected during the dry months. High phytoplankton growths lead to high photosynthetic activities thus enough food for organisms in higher trophic levels and for these algae. In addition, photosynthetic activities of the algae are usually higher during the dry months hence the present observation. Some of these algae are expected to die and decay. The decomposed matter will invariably increase the nutrients of this creek. The low $\mathrm{pH}$ makes nutrients (such as phosphate and nitrate) available to the primary producers.

The high abundance of phytoplankton in station 5 might be attributed to the large amounts of domestic and industrial wastes containing high level of phosphates from the Diobu and Eagle Island areas. The Diobu area of Port Harcourt is densely populated. The waterfront areas lack sanitary facilities.

The dominance of diatoms and blue-green algae indicate that Elechi Creek is polluted. Ruivo ${ }^{[13]}$ states that natural unpolluted environments are characterized by balanced biological conditions and contains a great diversity of plants and animals life's with no one species dominating. The difference in the community structure despite the dominance by diatoms is mainly due to the importance assumed by Cyanophyceae, Chlorophyceae and Euglenophyceae in the phytoplankton community. However, the distribution of diatoms reflects the average ecological conditions of this aquatic environment ${ }^{[14]}$. Dinoflagellates were the least abundant and this might be attributed to their inefficiency to compete for nutrients ${ }^{[15,16]}$.

The maximum number of diatoms species in station 5 might probably due to immense municipal wastes from the surroundings. The recorded dominant 
species could be as a result of high phosphate concentration and organic pollutants in these wastes. These species have been implicated with organic pollution. The same reason for highest number of diatom species in station 5 might be given for bluegreen algae species in station 5. Anabaena spiroides had also been implicated with organic pollution ${ }^{[17]}$. The presence of dominant Euglena species further indicates organic pollution. However, the presence of Ceratium furcas in stations 4 and 5 also shows organic pollution in Elechi Creek. Dominant species might indicate that these species love nutrients-rich environment. The presence of all these indicator phytoplankton species serves as a warning to the rise in nutrient capabilities of Elechi Creek. It is possible that diatoms and blue-green algae possess resilient ability to withstand organic pollution. Organic pollution eliminates the enemies of the more tolerant species which in turn increase in numbers.

The observed spatial variations of the phytoplankton might be attributed to the varied physico-chemical parameters. The recorded high temperature and low transparency favored the high abundance of phytoplankton. This is expected in tropical water bodies and fell within the acceptable range ${ }^{[18]}$. The present range of transparency is characteristic of brackish environment ${ }^{[18]}$. The recorded salinity, chloride and alkalinity were suitable for phytoplankton growths. The recorded salinity shows brackish environment. Salinity is one of the major factors influencing algae zonation and distribution within estuaries, both in terms of range of values and rate of changes ${ }^{[12]}$. It might be responsible for the observed variations of phytoplankton in this creek.

One of the factors that is likely to play an important role in determining community productive levels is nutrients availability; nitrogen, phosphate and sulphate $^{[12]}$. No station showed absence of nitrogen (nitrate-nitrogen) or phosphate (phosphate-phosphorus) but the concentrations seem limiting hence the varied diatoms density. This emphasizes the influence and significant role of nutrients in phytoplankton productivity in the stations. The phosphate level recorded was higher than the permissible concentrations in natural aquatic bodies $\left(0.10 \mathrm{mg} \mathrm{L}^{-1}\right)^{[19]}$. This might be attributed to the raw human and animal faces. The high sulphate concentration is characteristic of brackish water $^{[18]}$. However, the high nutrients levels (phosphate, sulphate and nitrogen-nitrate) enhanced the growths of phytoplankton.

It could reasonably be concluded that Elechi Creek is hypereutrophic and organic polluted. The high nutrients status favors the high abundance of phytoplankton. The municipal effluents (especially raw human and animal faces) discharges must be discouraged or discontinued. Detergents with low concentration of phosphate are recommended for manufacturing and use. Municipal wastes must be treated and/or recycled before discharge into this natural aquatic body. Therefore, a continuous environmental surveillance of this creek is advocated to keep its biological integrity.

\section{CONCLUSION}

The high abundance of phytoplankton in Elechi Creek can support fisheries but its nutrients availability especially phosphate of Elechi Creek is very high. This high phosphate level indicates that this creek is under stress. Its biological integrity may completely be destroyed if remedial and surveillance measures are not promptly taken by the Rivers State government.

\section{ACKNOWLEDGEMENT}

The researchers acknowledge all staff of the Institute of Pollution Studies, Rivers State University of Science and Technology, Port Harcourt, Rivers State, Nigeria especially Mr. U. Ikoro and Mr. H. Uyi for their technical assistance. This study was financially funded by the authors (personal savings).

\section{REFERENCES}

1. Townsend, C.R., J.L. Harper and M. Begon, 2000. Essentials of Ecology. 3rd Edn., Blackwell Science Publishers, London, ISBN: 1405103280, pp: 530.

2. Conde, D., S. Bonilla, L. Aubriot, R. de León and W. Pintos, 2007. Relative contribution of planktonic and benthic microalgae production in an eutrophic coastal lagoon of South America.

3. Wehr, J.D. and J.P. Descy, 1998. Use of phytoplankton in large river management. J. Phycol., 34: 741-749. DOI: 10.1046/j.15298817.1998.340741.x

4. Barnes, R S.K., 1980. Coastal Lagoons. 2nd Edn., Cambridge University Press, London, ISBN: 0521299454, pp: 106.

5. Wilcox, B.H., 1980. Angiosperm flora of the niger delta mangal. A taxonomic review. Proceedings of Workshop on the Niger Delta Mangrove Ecosystem, Oct. 19-21, Port Harcourt, pp: 19-23.

6. Eaton, A.D., L.S. Clesceri and A.E. Greenberg, 1999. Standard Methods for the Examination of Water and Wastewater, 20th Edn., American Public Health Association, McGraw-Hill Publishers, Baltimore, Maryland, Washington DC., ISBN: 10: 0875532357, pp: 1325. 
7. Boyd, C. E., 1981. Water Quality in Warmwater Fish Ponds. 2nd Edn., Craftmaster Publishers, Alabama, pp: 359.

8. Newell, G.E. and R.C. Newell, 1963. Marine Plankton. A Practical Guide. 1st Edn., Hutchinson Publishing Limited, London, ISBN: 13: 9780090692514, pp: 224.

9. Han, M., 1978. Illustration of Freshwater Plankton.1st Edn., Agricultural Press, Alabama, pp: 171.

10. Prescott, G.W., 1982. How to Know the Freshwater Algae. 3rd Edn., McGrawHill, Washington, DC., ISBN: 0697047547, pp: 304.

11. Kadiri, M.O., 1988. A taxonomic study of the genus Closterium (Nizch. 1919, Ralfs 1945) (desmichaceae chlorophyta) in small Nigeria reservoir with ecological notes. Trop. Freshwater Biol., 1: 71-90.

12. Frankovich, T.A., Gaiser, E.E., Zieman, J.C. and Wachnicka, A.H. 2006. Spatial and temporal distributions of epiphytic diatoms growing on Thalassia testudinum Banks ex Köńig: Relationships to water quality. Hydrobiologia, 569: 259-271.

http://cat.inist.fr/?aModele $=$ afficheN\&cpsidt $=18090281$

13. Ruivo, M.R., 1972. Marine Pollution and Sea Life. 1st Edn., Fishing News (Books) Publishers, England, pp: 630.
14. Passy, S.I., R.W. Bode, D.M. Carlson and M.A. Novak, 2004. Comparative Environmental Assessment in the Studies of Benthic Diatom, Mmacroinvertebrate and Fish Communities. Int. Rev. Hydrobiol., 89: 121-138. DOI: 10.1002/iroh.200310721

15. Boney, A.D., 1983. Phytoplankton. 4th Edn., Edward Arnold Publishers Limited, London, ISBN: 0713124768, pp: 115.

16. Reynold, C.S., 2006. The Ecology of Phytoplankton. 1st Edn., Cambridge Publishers, New York, ISBN: 0521844134, pp: 535.

17. Hallegraeff, G.M., D.M. Anderson and A.D. Cembella, 1995. Manual on Harmful Marine Microalgae. IOC Manual and Guides 33. UNESCO, France, pp: 551.

18. Asonye, C.C., N.P. Okolie, E.E. Okenwa and U.G. Iwuanyanwu, 2007. Some physico-chemical characteristics and heavy metal profiles of Nigerian rivers, streams and waterways. Afr. J. Biotechnol., 6: 617-624.

http://www.academicjournals.org/AJB/PDF/pdf200 7/5Mar/Asonye\%20et\%20al.pdf

19. McNeely, R.N., V.P. Neimanis and L. Dwyer, 1979. Water Quality Sourcebook. A guide to Water Quality Parameters. Inland Waters Directorate, Water Quality Branch, Ottawa, ISBN: 0662105206, pp: 89. 